\title{
A pilot study on the efficacy of local anaesthetics
}

\author{
Articaine and lignocaine efficiency in infiltration anaesthesia: a pilot study P. C. Oliveira, M. C. Volpato, \\ J. C. Ramacciato and J. Ranali Br Dent J 2001; 197: 45-46
}

\section{Aim}

The purpose of this study was to evaluate the onset of action of pulpal and soft tissue anaesthesia, and pain experience after buccal and palatal infiltrative injections with 4\% articaine with $1: 100,000$ adrenaline, and 2\% lignocaine with 1:100,000 adrenaline.

\section{Method}

A double blind cross-over study was conducted with 20 healthy adult subjects who, in two appointments at least two weeks apart, randomly received an infiltration anaesthesia with the solutions in the buccal and palatal regions of the upper right canine. The tooth was tested with a pulp tester before (to establish its baseline response), and after the injection, until return to the base threshold level. The pain experience caused by palatal injection was verified by the visual analogue scale (VAS). Data were analysed using Wilcoxons test $(\mathrm{a}=0.05)$.

\section{Results}

There were no significant statistical differences between the solutions with respect to VAS $(p=0.45)$, onset of action $(p=0.80)$ and pulpal $(p=0.08)$ and soft tissue $(p=0.18)$ anaesthesia duration, although pulpal anaesthesia may have reached statistical significance if a higher number of volunteers had been used.

\section{Conclusion}

Under the conditions of this study it can be concluded that both anaesthetic solutions showed similar pain experience.

\section{IN BRIEF}

- Both articaine and lignocaine show poor diffusion to the palate, causing pain during palatal injection. This paper provides information for giving patients the best, least painful injection without relying solely on the possible diffusion properties of the anaesthetic.

- A comparison between articaine and lignocaine gives important information about articaine.

- The relationship between price and benefit may be important in countries where there is a difference in cost between articaine and lignocaine.

\section{COMMENT}

Articaine is an amide local anaesthetic which was introduced into dentistry in the United Kingdom in 1998. It has been used in other countries for over 20 years. In some countries, such as Germany, articaine is the most popular local anaesthetic in use in dentistry. It is presented as a $4 \%$ solution. Some concern has been expressed at using this solution during regional block techniques such as inferior alveolar and lingual nerve block. ${ }^{1}$ This is because non-surgical paraesthesias of the lip and tongue have been associated with solutions using relatively high concentrations of local anaesthetics. ${ }^{2}$ Neurotoxicity is concentration dependent. ${ }^{3}$ It would appear, therefore that the main indication for using $4 \%$ articaine in dental practice is during infiltration anaesthesia. This double-blind cross-over study from Jose Ranali's team in Piracicaba compared $4 \%$ articaine with 1:100,000 epinephrine (adrenaline) and 2\% lidocaine (lignocaine) with 1:100,000 epinephrine as agents for infiltration anaesthesia in the maxillary canine region in 20 healthy adult volunteers. They investigated the onset time and duration of pulpal anaesthesia following buccal infiltration. They also studied the duration of perceived altered sensation in the lip post-injection. Finally, they recorded the discomfort of palatal infiltrations using the same solutions. They concluded that there were no statistically significant differences between the two solutions in any of the parameters investigated. They did, however, note a trend for pulpal anaesthesia to last longer after the use of the articaine solution. They found no evidence to support the suggestion that buccal infiltration of articaine provides palatal anaesthesia.

The results of this study suggest that both $4 \%$ articaine and $2 \%$ lidocaine, when combined with 1:100,000 epinephrine, provide pulpal anaesthesia after buccal infiltration in the maxillary canine region in adult volunteers. Both solutions produce similar degrees of discomfort when injected into palatal mucosa.

J. G. Meechan, Senior Lecturer, School of Dental Sciences, University of Newcastle upon Tyne doi:10.1038/sj.bdj.4811419

1. Pedlar J. Prolonged paraesthesia. Br Dent J2003 194:181.

2. Haas D A Lennon D. A 21 year retrospective study of reports of paraesthesia following local anesthetic administration. J Can Dent Assoc 1995 61:319-330.

3. Johnson M E, Saenz J A, DaSilva A D, Uhl B, Gores G J. Effect of local anesthetic on neuronal cytoplasmic calcium and plasma membrane lysis (necrosis) in a cell culture model. Anesthesio/ 2002 97: 1466-1476. 